\title{
Use of chimeric antigen receptor T cells in allogeneic hematopoietic stem cell transplantation
}

\author{
Jun Liu ${ }^{1}$, Xi Zhang ${ }^{1}$, Jiang F Zhong ${ }^{2}$ \& Cheng Zhang*,1 \\ ${ }^{1}$ Department of Hematology, Xinqiao Hospital, Army Medical University, Chongqing, People's Republic of China \\ ${ }^{2}$ Division of Periodontology, Diagnostic Sciences \& Dental Hygiene, \& Division of Biomedical Sciences, Herman Ostrow School of \\ Dentistry, University of Southern California, Los Angeles, CA, USA \\ *Author for correspondence: Tel.: +86 0236877 4209; chzhang2014@163.com
}

The chimeric antigen receptor T (CAR-T) cells play an antileukemia role, and can be used to treat or prevent relapse by targeting minimal residual disease for patients undergoing allogeneic hematopoietic stem cell transplantation (allo-HSCT). However, the infusion of allogeneic CAR-T cells may also cause graftversus-host disease, which limited their applications during and after allo-HSCT. In this review, we discuss the clinical trials that applying CAR-T cells before allo-HSCT and the use of donor-derived CAR-T cells as conditioning regimen during allo-HSCT. At last, we analyzed the effect of donor-derived CAR-T cells on preventive infusion after allo-HSCT.

First draft submitted: 29 June 2017; Accepted for publication: 25 October 2018; Published online: 7 December 2018

Keywords: acute lymphoblastic leukemia $\bullet$ acute myeloid leukemia $\bullet$ allogeneic CAR-T cells $\bullet$ allogeneic hematopoietic stem cell transplantation • CAR-T cells $\bullet$ conditioning regimen • donor-derived CAR-T cells • hematological malignancies $\bullet$ preventive infusion

The use of chimeric antigen receptor T (CAR-T) cells is an excellent example of applying basic research concept to clinical treatment. The CAR-T cells have shown a strong function in antileukemia [1,2]. With the advancement of technology, there are four generations of CAR-T cells available now [3]. At present, the second generation is extensively used in the treatment of hematological malignancies.

Allogeneic hematopoietic stem cell transplantation (allo-HSCT) is one of the most effective methods to treat refractory/relapsed acute lymphoblastic leukemia (ALL) [4]. The high relapse rate has been the main cause of death for patients with ALL after allo-HSCT [5,6]. And how to decrease the relapse rate after allo-HSCT is an important matter in treating ALL. Applications of CAR-T cells have resulted in good outcomes in refractory/relapsed ALL [7]. However, the adverse events, especially cytokine release syndrome (CRS), still need to be addressed for the application of CAR-T cells in clinical treatments [8-10]. CAR-T cells can treat or prevent relapse by targeting minimal residual disease (MRD) for B-cells malignancies. However, the infusion of allogeneic CAR-T cells could also cause the graft-versus-host disease (GVHD), which limits the use of CAR-T cells during and after alloHSCT. The outcome is very poor for patients with refractory/relapsed hematological malignancies undergone allo-HSCT without reaching complete remission (CR) [11]. In this review, we discuss using CAR-T cells with allo-HSCT for treating refractory/relapsed B-cells malignancies, especial for B-cell ALL. Further, we discuss the use of donor-derived CAR-T cells as a part of conditioning regimen during allo-HSCT. Finally, we discuss the role of donor-derived CAR-T cells on preventive infusion after allo-HSCT for preventing relapse and improving long-term survival of patients.

\section{Production of allogeneic CAR-T cells}

The production of allogeneic CAR-T cells needs several steps. The first step is leukapheresis of leukocytes from the donors. Then, the T cells are separated from the leukocytes [12]. The third step is the separation of CD4/CD8 T-cell subsets by using specific markers or antibody-bead conjugates. Then, the T cells were activated by co-culturing 
with anti-CD3/anti-CD28 monoclonal antibodies coated beads; autologous antigen-presenting cells from donors; anti-CD3 antibodies in combination with feeder cells and growth factors, such as IL-2 [13,14].

\section{CAR-T cells as a bridge of allogeneic transplantation}

Many studies showed that using CAR-T cells can achieve approximately $70-90 \%$ CR in relapsed/refractory B-cell malignancies [15-20]. However, CAR-T cells only can survive in the body for a short period of time, some of the patients relapsed quickly [21,22]. In our center, a total of 14 patients with refractory/relapsed B-ALL were treated with CD19-CAR-T cells. Among them, seven cases use autologous CAR-T cells and seven cases use cells from the donors. Eight patients achieved CR, but three of these patients relapsed within 3 months (Zhang C et al., UnPUBlished DATA). It is noteworthy that we have treated a case of B-ALL patients with CNS recurrence. After the treatment of CAR-T cells, the patient remains negative for CNS leukemia. Only one patient died on day 10. The main adverse reactions were granulocyte deficiency, anemia, thrombocytopenia and thrombocytopenia. Thus, further studies should be carried out to improve the long-term outcomes.

Some researchers showed that CAR-T cells treatment with allo-HSCT may be the best way to treat relapsed/refractory B-cell malignancies. Zhang et al. had showed that the patients with relapsed/refractory Bcell malignancies showed no sign of relapse if they received CAR-T cells treatment followed by allo-HSCT at the last follow-up [23]. In personal communication, Tong also showed that the survival is better for patients with refractory/relapsed B-cells ALL who received allo-HSCT after CD19-CAR-T cells treatment compared with the patients only received CAR-T cells [24].

However, it is controversial whether patients with refractory/relapsed B-cell malignancies should receive alloHSCT after CAR-T cells. A recent study showed that there is no significant difference between patients who underwent allo-HSCT and those who did not (79 vs $80 \%$ ) after CAR-T cells treatment in terms of 6-month overall survival [25]. The 6-months overall survival rates were $64 \%$ in patients who did not receive allo-HSCT after CD19-CAR-T cells treatment, and 70\% in patients who underwent allo-HSCT post-CAR-T cells treatment without statistical difference.

A few studies showed good outcomes for relapsed/refractory B-cell malignancies for those who received CAR-T cells treatment followed by allo-HSCT. However, there are some concerns on these studies. First, all of these studies were not randomized clinical trials and the follow-up time is short, along with a small case number, thus make it difficult to draw the definitive conclusions. Second, the controlled trials should be performed to compare the outcomes of CAR-T cells treatment followed with or without allo-HSCT for a longer follow-up time.

\section{CAR-T cells as conditioning regimen}

After allo-HSCT, disease relapse remains a significant cause of treatment failure in acute leukemia [26]. It is crucial to eliminate the leukemia cells as much as possible during allo-HSCT. Although myeloablative conditioning can eliminate most leukemia cells, procedure-related toxicities restrict its use, especially for older and weak patients with high leukemia burden $[27,28]$. Reduced-intensity conditioning allo-HSCT has less procedure-related toxicities and provides adequate immunosuppression. But the relapse incidence remains high [29-32]. Thus, it is vital to know how to further eliminate MRD and prevent the relapse in these patients.

Although the CAR-T cells have been used in relapsed/relapse B cells malignancies with good outcomes, the outcome is still poor for nonresponse patient treated by donor lymphocyte infusion (DLI) after allo-HSCT [33]. The outcome is also poor for a patient with a higher burden of disease [34]. In addition, it is very difficult to collect sufficient $\mathrm{T}$ cells for the construction of CAR-T cells from patients who have an extremely high leukemia burden $[23,35]$.

One study showed that the donor-derived anti-CD123-41BB-CAR-T cells have graft-versus-leukemia (GVL) effect after infusion in an acute myeloid leukemia xenograft model with NSG mice [36]. In this study, the CAR was constructed with a third-generation self-inactivating lentiviral vector plasmid, pRRL-SIN-CMV-eGFP-WPRE, in which the cytomegalovirus promoter was replaced by the EF- $1 \alpha$ promoter. The study showed that CAR-T cells have GVL effect [36]. In another murine haploidentical HSCT model, donor-derived CD19-CAR-T cells constructed with retroviral vector can induce GVL effect and diminish GVHD effect at day 1 [37].

A recent study systematically analyzed GVL effect and GVHD complication for 72 patients with relapsed ALL after allo-HSCT and received donor-derived CAR-T cells [38]. In this study, only 5 of 72 patients (6.9\%) developed slight GVHD without other serious side effects such as CRS and CNS toxicity. The authors indicated that donor-derived CAR-T cells are highly effective for relapse prophylaxis, salvaging relapse or MRD clearance. 
Table 1. Clinical trial registrations on allogeneic chimeric antigen receptor $\mathrm{T}$ cell in allogeneic transplantation.

\begin{tabular}{|c|c|c|c|c|}
\hline Center & Clinical trial number & Disease & CAR-T type & Status \\
\hline $\begin{array}{l}\text { Xinqiao Hospital, Army Medical } \\
\text { University, Chongqing, China }\end{array}$ & ChiCTR1800015353 & ALL & CD19 & Recruiting \\
\hline $\begin{array}{l}\text { Affiliated Hospital of Xuzhou } \\
\text { Medical University, China }\end{array}$ & ChiCTR-OIC-17012374 & $\begin{array}{l}\text { B-cell hematologic } \\
\text { malignancies }\end{array}$ & CD19 & Recruiting \\
\hline $\begin{array}{l}\text { Xinqiao Hospital, Army Medical } \\
\text { University, Chongqing, China }\end{array}$ & ChiCTR-OOC-16008447 & ALL & CD19 & Recruiting \\
\hline $\begin{array}{l}\text { Fred Hutchinson Cancer } \\
\text { Research Center }\end{array}$ & NCT01475058 & ALL & CD19 & Completed \\
\hline $\begin{array}{l}\text { Affiliated Hospital to Academy } \\
\text { of Military Medical Sciences, } \\
\text { Beijing Shi, China }\end{array}$ & NCT03114670 & Adult AML & 41BB- CD123 & Recruiting \\
\hline $\begin{array}{l}\text { Biotherapeutic Department } \\
\text { and Hematology Department } \\
\text { of Chinese PLA General } \\
\text { Hospital, Beijing, China }\end{array}$ & NCT03463928 & B-cell leukemia & Bispecific CD19/22 or CD19 & Recruiting \\
\hline National Cancer Institute, USA & NCT01087294 & B-cell cancer & CD19 & Recruiting \\
\hline University of Pennsylvania, USA & NCT01551043 & ALL & CD19 & Completed \\
\hline
\end{tabular}

The trials are registered at www.chictr.org.cn/ and ClinicalTrials.gov. Search performed on 3rd September 2018.

ALL: Acute lymphoblastic leukemia; AML: Acute myeloid leukemia; CAR-T: Chimeric antigen receptor T cell.

Table 2. Chimeric antigen receptor T cells used as conditioning regimens and preventive infusion after allogeneic hematopoietic stem cell transplantation.

\begin{tabular}{llllllll}
\hline Center & Target & No. & Disease & $\begin{array}{l}\text { Dose of CAR-T } \\
\text { cells }\end{array}$ & $\begin{array}{l}\text { Role of CAR-T } \\
\text { cells }\end{array}$ & $\begin{array}{l}\text { SVHD } \\
\text { follow-up }\end{array}$ \\
$\begin{array}{l}\text { Department of } \\
\text { hematology, }\end{array}$ & CD19 & 3 & ALL & $6.4 \times 10^{6} / \mathrm{kg}$ & $\begin{array}{l}\text { Conditioning } \\
\text { regimens }\end{array}$ & No & No
\end{tabular}

Xinqiao

Hospital, Army

Medical

University,

Chongqing,

China

\begin{tabular}{|c|c|c|c|c|c|c|c|c|}
\hline $\begin{array}{l}\text { Center for Cell } \\
\text { and Gene } \\
\text { Therapy, Baylor } \\
\text { College of } \\
\text { Medicine, } \\
\text { Houston, Texas, } \\
\text { USA }\end{array}$ & CD19 & 2 & ALL & $\begin{array}{l}9.7 \times 10^{7} \text { and } \\
5.8 \times 10^{7} \\
\text { respectively }\end{array}$ & $\begin{array}{l}\text { Preventive } \\
\text { infusion }\end{array}$ & No & No & $\begin{array}{l}2 \text { weeks to } 8 \\
\text { months }\end{array}$ \\
\hline $\begin{array}{l}\text { The University } \\
\text { of Texas MD } \\
\text { Anderson } \\
\text { Cancer Center, } \\
\text { Houston, Texas, } \\
\text { USA }\end{array}$ & CD19 & 19 & $\begin{array}{l}\text { B-cell } \\
\text { malignancies }\end{array}$ & $\begin{array}{l}10^{6} / \mathrm{m}^{2} \text { to } \\
10^{8} / \mathrm{m}^{2}\end{array}$ & $\begin{array}{l}\text { Preventive } \\
\text { infusion }\end{array}$ & $\begin{array}{l}\text { Three patients } \\
\text { with GVHD. One } \\
\text { died from liver } \\
\text { failure }\end{array}$ & No & 12-month OS: $63 \%$ \\
\hline
\end{tabular}

Therefore, the donor-CAR-T cells may be safe and effective in allo-HSCT. Recently, Ai et al. reported an older patient with refractory ALL, who was treated with low numbers of donor-derived CD19-CAR-T cells and with low dose of recombinant human granulocyte colony-stimulating factor-mobilized peripheral blood stem cells [35]. This patient developed serious GVHD and by treating with anti-GVHD drugs, the related complication was controlled. However, this patient died at last because of severe infection on day 31. It is still not clear that whether donor-derived CAR-T cells should be used during allo-HSCT as a part of conditioning regimens because of the related complications, such as CRS and GVHD, especially for patients with high leukemia burden who can collect enough $\mathrm{T}$ cell for the construction of CAR-T cells. In our center, a 12-year-old girl with refractory/relapsed ALL was treated with donor-derived CD19-CAR-T cells as a reduced-intensity conditioning regimen for haploidentical transplantation [39]. This patient received a reduced-intensity conditioning regimen, then infusion with equal dose 
of the donor-derived CD19-CAR-T cells for 4 days infusion with total dose of $6.4 \times 10^{6} / \mathrm{kg}$. The granulocytemobilized peripheral blood stem cells and granulocyte colony-stimulating factor-mobilized bone marrow was infused on days 1 and 2, respectively. The tacrolimus and microphenolate motetil were used on day 1 , and the antithymocyte globulin (ATG) was used on days +14 and +15 . The highest level of the donor-derived CD19CAR-T cells in the peripheral blood was reached on day 8 and gradually dropped until undetectable on day +40 . The patient completely engrafted without serious complications and is disease free at the last follow-up.

Although it is easier to get sufficient $\mathrm{T}$ cells from healthy donor in construction of CAR-T cells, the proliferation rate of allogeneic CAR-T cells is slower than that of the autologous CAR-T cells. Therefore, it must be careful when using the immunosuppressors because of their potential effect in killing CAR-T cells and inhibiting the proliferation of CAR-T cells during transplantation. In this study, the immunosuppressors of mycophenolate mofetil and tacrolimus were changed from day 7 to day 1 after the 7 th day of the first infusion of CAR-T cells, which was adopted to avoid or alleviate the killing effect and the inhibition of the proliferation of the CAR-T cells [39]. The ATG) can also kill and inhibit the proliferation of CAR-T cells. In this protocol, the ATG was used only two-times on days +14 and +15 after 2 weeks of the infusion of CAR-T cells. Then we further applied this treatment in another two patients, which also showed good outcomes (Zhang C et al., Unpublished Data). Nevertheless, a trial with larger number of patients is still needed to confirm the curative effect of CAR-T cells.

\section{CAR-T cells in preventing relapse after transplantation}

The relapse rate has been high for patients with ALL. Even with allo-HSCT, the long-term survival is still poor for patients with refractory/relapsed ALL [40-42]. So it is vital to prevent relapse for patients with ALL after allo-HSCT.

One of the main methods used to prevent or treat relapse for patients with ALL after allo-HSCT is DLI through the GVL effect [43]. Severe acute GVHD developed in approximately a third of DLI [44]. Besides, the curative effect is very limited for relapse ALL after allo-HSCT or with preventive DLI infusion after transplantation [45-47]. Preventing relapse for patients with ALL remains an unmet clinical challenge after allo-HSCT. Therefore, new treatment strategies are still needed.

Although allogeneic CAR-T cells have been successfully used on patients with refractory/relapsed ALL without serious complications, the long-term survival is still needed to be improved [48]. Pre-emptive therapy should be performed upon the detection of MRD via molecular or immunophenotypic methods [49]. Better detection of MRD can be developed with more advanced molecular analyses, such as single-cell technology and microfluidic devices [50,51]. As a new method of immunotherapy, CAR-T cells could be applied for preventive infusion after allo-HSCT. Recent study showed that between days +55 and +200 after allo-HSCT is the best time for immunotherapy [23].

The infused donor-derived virus-specific $\mathrm{T}$ cells expanded in vivo can persist long term and display antiviral activity without GVHD after allo-HSCT [52-54]. Therefore, Dotti et al. used the donor-derived CD19.CAR-virusspecific T cells to treat B-cell malignancies in a Phase I study in 2003 [55]. In this study, one patient remains in CR for $>8$ months after CD19-CAR-T cells infusion, and another patient remains in CR for 8 weeks after CAR-T cells treatment. The two patients did not develop GVHD and serious CRS. In this study, whether the patients received immunosuppressors after CAR-T cells infusion is unclear.

Cooper et al. reported a Phase I clinical trial for 19 patients with B-cell malignancies who received a CD19CAR-T cells infusion after allo-HSCT [56]. The CAR-T cells were constructed using the Sleeping Beauty transposon/transposase system to express a CD19-specific CAR. The tacrolimus and minidose methotrexate were used as GVHD prophylaxis for matched-sibling HSCT, and tacrolimus, mycophenolate mofetil and post cyclophosphamide were used for haploidentical HSCT. The drugs of GVHD prophylaxis were tapered and discontinued 6 months after allo-HSCT. The CAR-T cells were administered during 6-12 weeks after allo-HSCT based on a median time occurrence of acute GVHD is 6 weeks after allo-HSCT. The white blood cells in most of these patients were normal $\left(1.2 \times 10^{9} / 1\right.$ to $\left.9.2 \times 10^{9} / \mathrm{l}\right)$ when performed CAR-T cells infusion. The numbers of CAR-T cells were $10^{6} / \mathrm{m}^{2}$ to $10^{8} / \mathrm{m}^{2}$. The CAR-T cells persisted in vivo for 51 days in average, and in one patient, the CAR-T cells still can be detected even after 1 year. No acute infusion or delayed toxicities were developed. Three patients developed GVHD. One of them with grade 1 acute skin GVHD was resolved with topical steroids, one with chronic skin GVHD responded to systemic steroids, and one patient died from liver failure with a component of liver GVHD due to a prior history of drug-induced liver toxicity. The 12-month progression-free and overall survivals rates were 53 and $63 \%$, respectively. This study suggested that CAR-T cells infusion is safe and effective, and the immunosuppressors did not affect the proliferation of CAR-T cells and may inhibit the release of cytokines. 
In our center, we performed a prospective clinical trial to explore the safety and efficacy of preventive infusion with donor-derived CD19.CAR-T after allo-HSCT at day +60 (ChiCTR1800015353). In this study, the GVHD prophylaxis was continually used according to the standard protocol of allo-HSCT. Our preliminary results showed that the CAR-T cells persisted, and they are safe and effective without serious adverse effects, such as CRS and GVHD. The reason that the infusion of allogeneic CAR-T cells post-transplant seldom induced GVHD may be the continual use of immunosuppressor, and that the donor-derived CAR-T cells are partial tolerance forms because the immune system origins from the donors after hematopoietic reconstitution with full chimerism, which is similar to the patients' autologous CAR-T cells. At present, there are several clinical trials on the use of allogeneic CAR-T cells (Table 1).

\section{Conclusion}

Some questions still need to be solved for the use of allogeneic CAR-T cells. The sequential CAR-T cells or dual CAR-T cells should be deeply explored to escape off-target effect and further improve the curative effect and long-term survival. Universal CAR-T cells may be ideal CAR-T cells in the future. New CAR-T cells those can combat relapse, mitigate overactivation and enhance specificity should be produced.

Altogether, the application of CAR-T cells before allo-HSCT showed good outcome for refractory/relapsed Bcells malignancies, suggesting a safe and effective role of CAR-T cells as conditioning regimens (Table 2). However, further randomized and double-blinded clinical trials should be performed to systematically evaluate the role of CAR-T cells in conditioning regimens and preventive infusion after allo-HSCT.

\section{Future perspective}

Although allogeneic or donor-derived CAR-T cells showed good outcomes at present, there are still some questions need to be solved. First, the sequential CAR-T cells or dual CAR-T cells such as CD19CD22 CAR-T cells, CD5CD19 CAR-T cells and CD33CD123 CAR-T cells should be deeply explored in order to escape off-target effect and increase long-term survival rate with improved curative effect. Second, although the use of donor-derived allogeneic CAR-T cells showed good outcomes during and after transplantation with little complications, the production period of CAR-T cells is still too long; the universal CAR-T cells that can be used immediately may be one of the best CAR-T cells in future. Third, the new CAR-T cells that can combat relapse, mitigate overactivation and enhance specificity should be developed. A recent study reported a split, universal and programmable CAR system, which simultaneously encompasses multiple critical 'upgrades', such as finely tune T-cell activation strength, sense and logically respond to multiple antigens, and the ability to switch targets without re-engineering the $T$ cells [57]. Due to the persistence and engraftment related to the state of T-cell differentiation, limiting differentiation

\section{Executive summary}

\section{Background}

- Relapse is still the main factor of death after allogeneic hematopoietic stem cell transplantation (allo-HSCT).

- At present, there are no effective methods to prevent the relapse of disease after allo-HSCT.

- The chimeric antigen receptors T (CAR-T) cells can treat or prevent relapse by targeting minimal residual disease for B cells malignancies; however, whether the CAR-T cells can be safely be used with allo-HSCT is still unclear.

Production of allogeneic CAR-T cells

- The production of allogeneic CAR-T cells needs several steps.

- The allegeneic CAR-T cells are usually produced from the donors.

CAR-T cells as bridge of allogeneic transplantation

- CAR-T cells can make a good outcome for refractory/relapsed B cells malignancies; however, relapse rate is very high.

- It may be the best way to treat relapsed/refractory acute lymphoblastic leukemia with CAR-T cells followed by allo-HSCT.

CAR-T cells as conditioning regimen

- CAR-T cells can be used as a part of conditioning regimens in allo-HSCT.

- The immunosuppressors did not affect the proliferation of CAR-T cells and may inhibit the release of cytokines.

CAR-T cells in preventing relapse after transplantation

- It is safe and effective for preventive infusion of CAR-T cells after allo-HSCT.

- The immunosuppressors did not affect the proliferation of CAR-T cells and may inhibit the release of cytokines by CAR-T cells. 
may enhance the efficacy of CAR-T cell therapy. A recent study showed that producing CAR-T cells with less time can improve effector function [58].

\section{Financial \& competing interests disclosure}

This study was funded by the clinical key foundation of Xinqiao Hospital of Army Military Medical University (No. 2016YLC03). The authors have no other relevant affiliations or financial involvement with any organization of entity with a financial interest in or conflict with the subject matter or materials discussed in the manuscript apart from those disclosed. No writing assistance was utilized in the production of this manuscript.

\section{References}

Papers of special note have been highlighted as: $\bullet$ of interest; $\bullet \bullet$ of considerable interest

1. Brentjens RJ, Davila ML, Riviere I et al. CD19-targeted T cells rapidly induce molecular remissions in adults with chemotherapy-refractory acute lymphoblastic leukemia. Sci. Transl. Med. 5(177), 177ra38 (2013).

2. Kochenderfer JN, Dudley ME, Kassim SH et al. Chemotherapy-refractory diffuse large B-cell lymphoma and indolent B-cell malignancies can be effectively treated with autologous T cells expressing an anti-CD19 chimeric antigen receptor. J. Clin. Oncol. 33(6), 540-549 (2015).

3. Zhang C, Liu J, Zhong JF, Zhang X. Engineering CAR-T cells. Biomark. Res. 5, 22 (2017).

4. Wu S, Zhang C, Zhang X, Xu YQ, Deng TX. Is peripheral blood or bone marrow a better source of stem transplantation in cases of HLA-matched unrelated donors? Crit. Rev. Oncol. Hematol. 96(1), 20-33 (2015).

5. Lussana F, Intermesoli T, Gianni F et al. Achieving molecular remission before allogeneic stem cell transplantation in adult patients with Philadelphia chromosome-positive acute lymphoblastic leukemia: impact on relapse and long-term outcome. Biol. Blood Marrow Transplant. 22(11), 1983-1987 (2016).

6. Lou Y, Ma Y, Li C et al. Efficacy and prognostic factors of imatinib plus CALLG2008 protocol in adult patients with newly diagnosed Philadelphia chromosome-positive acute lymphoblastic leukemia. Front. Med. 11(2), 229-238 (2017).

7. Park JH, Rivière I, Gonen M et al. Long-term follow-up of CD19 CAR therapy in acute lymphoblastic leukemia. N. Engl. J. Med. 378(5), 449-459 (2018).

8. Lee DW, Gardner R, Porter DL et al. Current concepts in the diagnosis and management of cytokine release syndrome. Blood 124(2), 188-195 (2014).

9. Maude SL, Frey N, Shaw PA et al. Chimeric antigen receptor T cells for sustained remissions in leukemia. N. Engl. J. Med. 37(16), 1507-1517 (2014).

10. Teachey DT, Lacey SF, Shaw PA et al. Identification of predictive biomarkers for cytokine release syndrome after chimeric antigen receptor T-cell therapy for acute lymphoblastic leukemia. Cancer Discov. 6(6), 664-679 (2016).

-. Constructes a model accurately predicting patients who received chimeric antigen receptors $T$ (CAR-T) cells treatment are likely to develop severe cytokine release syndrome before they become critically ill, which may guide future cytokine-directed therapy.

11. Wolach O, Amitai I, DeAngelo DJ. Current challenges and opportunities in treating adult patients with Philadelphia-negative acute lymphoblastic leukaemia. Br. J. Haematol. 179(5), 705-723 (2017).

12. Lee G, Arepally GM. Anticoagulation techniques in apheresis: from heparin to citrate and beyond. J. Clin. Apher. 27(3), 117-125 (2012).

13. Guedan S, Chen X, Madar A et al. ICOS-based chimeric antigen receptors program bipolar TH17/TH1 cells. Blood 124(7), 1070-1080 (2014).

14. McGarrity GJ, Hoyah G, Winemiller A et al. Patient monitoring and follow-up in lentiviral clinical trials. J. Gene Med. 15(2), 78-82 (2013).

15. Lee D, Kochenderfer J, Stetler-Stevenson M et al. T cells expressing CD19 chimeric antigen receptors for acute lymphoblastic leukaemia in children and young adults: a Phase I dose-escalation trial. Lancet 385(9967), 517-528 (2014).

16. Grupp SA, Maude SL, Shaw PA et al. Durable remissions in children with relapsed/refractory ALL treated with $\mathrm{T}$ cells engineered with a CD19-targeted chimeric antigen receptor (CTL019). Blood 126, 681 (2015).

17. Turtle CJ, Hanafi LA, Berger C et al. CD19 CAR-T cells of defined $\mathrm{CD}^{+}: \mathrm{CD}^{+}$composition in adult B cell ALL patients. J. Clin. Invest. 126(6), 2123-3218 (2016).

18. Magnani CF, Biondi A, Biagi E. Donor-derived CD19-targeted T cells in allogeneic transplants. Curr. Opin. Hematol. 22(6), 497-502 (2015).

19. Zuo YX, Wang JB, Lu AD et al. Chimeric antigen receptors $T$ cells in treatment of a relapsed pediatric acute lymphoblastic leukemia, relapse after allogenetic hematopoietic stem cell transplantation: case report and review of literature review. Zhonghua Xue Ye Xue Za Zhi 37(2), 115-118 (2016).

20. Dong L, Chang LJ, Gao Z et al. Chimeric antigen receptor 4SCAR19-modified T cells in acute lymphoid leukemia: a Phase II multi-center clinical trial in China. Blood 126, 3774 (2015). 
21. Zhang T, Cao L, Xie J et al. Efficiency of chimeric antigen receptor-modified T cells for treatment of B cell malignancies in Phase I clinical trials: a meta-analysis. Oncotarget 6(32), 33961-33971 (2015).

22. Maude S, Barrett DM. Current status of chimeric antigen receptor therapy for haematological malignancies. Br. J. Haematol. 172(1), $11-22(2016)$

23. Liu J, Zhong JF, Zhang X, Zhang X. Allogeneic CD19-CAR-T cell infusion after allogeneic hematopoietic stem cell transplantation in B cell malignancies. J. Hematol. Oncol. 10(1), 35 (2017).

-• Systematically reviews the use of allogeneic CAR-T cells after allogeneic hematopoietic stem cell transplantation (allo-HSCT). The allogeneic CAR-T cells are safe and effective for relapsed patients after allo-HSCT.

24. Pan J, Yang JF, Deng BP et al. High efficacy and safety of low-dose CD19-directed CAR-T cell therapy in 51 refractory or relapsed B acute lymphoblastic leukemia patients. Leukemia 31(12), 2587-2593 (2017).

-• Shows that no relapse was found in patients with the refractory/relapsed B-cells acute lymphoblastic leukemia (ALL)-received CAR-T cells following allo-HSCT.

25. Park JH, Riviere I, Wang X et al. Implications of minimal residual disease negative complete remission (MRD-CR) and allogeneic stem cell transplant on safety and clinical outcome of CD19-targeted 19-28z CAR modified T cells in adult patients with relapsed, refractory B-cell ALL. Blood 126, 682 (2015).

-• Analyzes the outcome on minimal residual disease (MRD) status post-CAR-T treatment followed by all-HSCT or not for refractory/relapsed ALL patients. The results show no differences in outcomes between the MRD' complete remission (CR) and $\mathrm{MRD}^{+} \mathrm{CR}$ cohorts regardless of whether they received allo-HSCT. However, the cases were small and the follow-up time was short.

26. Craddock C, Hoelzer D, Komanduri KV. Current status and future clinical directions in the prevention and treatment of relapse following hematopoietic transplantation for acute myeloid and lymphoblastic leukemia. Bone Marrow Transplant.doi:10.1038/s41409-018-0203-8 (2018) (Epub ahead of print).

27. Dhere V, Edelman S, Waller EK et al. Myeloablative busulfan/cytoxan conditioning versus reduced-intensity fludarabine/melphalan conditioning for allogeneic hematopoietic stem cell transplant in patients with acute myelogenous leukemia. Leuk. Lymphoma 59(4), 837-843 (2018).

28. Zeng W, Huang L, Meng F et al. Reduced-intensity and myeloablative conditioning allogeneic hematopoietic stem cell transplantation in patients with acute myeloid leukemia and myelodysplastic syndrome: a meta-analysis and systematic review. Int. J. Clin. Exp. Med. 7(11), 4357-4368 (2014).

29. Blaise D, Vey N, Faucher C, Mohty M. Current status of reduced-intensity-conditioning allogeneic stem cell transplantation for acute myeloid leukemia. Haematologica 92, 533-541 (2007).

30. Leonard JT, Hayes-Lattin B. Reduced intensity conditioning allogeneic hematopoietic stem cell transplantation for acute lymphoblastic leukemia; current evidence, and improving outcomes going forward. Curr. Hematol. Malig. Rep.doi:10.1007/s11899-018-0462-x (2018) (Epub ahead of print).

31. Mohty M, Labopin M, Volin L et al. Reduced-intensity versus conventional myeloablative conditioning allogeneic stem cell transplantation for patients with acute lymphoblastic leukemia: a retrospective study from the European Group for Blood and Marrow Transplantation. Blood 116(22), 4439-4443 (2010).

32. Bachanova V, Marks DI, Zhang MJ et al. $\mathrm{Ph}+$ ALL patients in first complete remission have similar survival after reduced intensity and myeloablative allogeneic transplantation: impact of tyrosine kinase inhibitor and minimal residual disease. Leukemia 28(3), 658-665 (2014).

33. Chen Y, Cheng Y, Suo P et al. Donor-derived CD19-targeted T cell infusion induces minimal residual disease-negative remission in relapsed B-cell acute lymphoblastic leukaemia with no response to donor lymphocyte infusions after haploidentical haematopoietic stem cell transplantation. Br. J. Haematol. 179(4), 598-605 (2017).

34. Haji-Fatahaliha M, Hosseini M, Akbarian A et al. CAR-modified T-cell therapy for cancer: an updated review. Artif. Cells Nanomed. Biotechnol. 44(6), 1339-1349 (2016).

35. Cai B, Guo M, Wang Y et al. Co-infusion of haplo-identical CD19-chimeric antigen receptor T cells and stem cells achieved full donor engraftment in refractory acute lymphoblastic leukemia. J. Hematol. Oncol. 9(1), 131 (2016).

36. Gill S, Tasian SK, Ruella $M$ et al. Preclinical targeting of human acute myeloid leukemia and myeloablation using chimeric antigen receptor-modified T cells. Blood 123(15), 2343-2354 (2014).

37. Ghosh A, Smith M, James SE et al. Donor CD19 CAR T cells exert potent graft-versus-lymphoma activity with diminished graft-versus-host activity. Nat. Med. 23(2), 242-249 (2017).

-. First found that the donor-derived CAR-T cells infused at the time of the allograft injection have graft-versus-lymphoma effect and diminished graft-versus-host disease effect in murine haploidentical HSCT model.

38. Anwer F, Shaukat AA, Zahid U et al. Donor origin CAR T cells: graft versus malignancy effect without GVHD, a systematic review. Immunotherapy 9(2), 123-130 (2017). 
39. Zhang C, Kong PY, Li S et al. Donor-derived CAR-T cells serve as a reduced-intensity conditioning regimen for haploidentical stem cell transplantation in treatment of relapsed/refractory acute lymphoblastic leukemia: case report and review of the literature. J. Immunother. J. Immunother. 41(6), 306-311 (2018).

-• The first paper to report the use of CAR-T cells as a conditioning regimen in haploidentical stem cell transplantation for patients with relapsed/refractory acute lymphoblastic leukemia. The patients successfully engraft without graft-versus-host disease and no serious complications. The CAR-T cells persist in the body for about 1 month.

40. Bhojwani D, Pui CH. Relapsed childhood acute lymphoblastic leukaemia. Lancet Oncol. 14(6), e205-e217 (2013).

41. Gao L, Zhang C, Gao L et al. Favorable outcome of haploidentical hematopoietic stem cell, transplantation in Philadelphia chromosome-positive acute lymphoblastic leukemia: a multicenter study in southwest China. J. Hematol. Oncol. 8(1), 90 (2015).

42. Chen XH, Zhang C, Zhang X et al. Role of antithymocyte globulin and granulocyte-colony stimulating factor-mobilized bone marrow in allogeneic transplantation for patients with hematologic malignancies. Biol. Blood Marrow Transplant. 15(2), 266-273 (2009).

43. Wang YX, Li YH. Efficacy of donor lymphocyte infusion for treating relapsed high-risk leukemia patients after allogeneic hematopoietic stem cell transplantation. Zhongguo Shi Yan Xue Ye Xue Za Zhi 23(4), 982-988 (2015).

44. Roddie C, Peggs KS. Donor lymphocyte infusion following allogeneic hematopoietic stem cell transplantation. Expert Opin. Biol. Ther. 11(4), 473-487 (2011).

45. Castagna L, Sarina B, Bramanti S, Perseghin P, Mariotti J, Morabito L. Donor lymphocyte infusion after allogeneic stem cell transplantation. Transfus. Apher. Sci. 54(3), 345-355 (2016).

46. Rambaldi A, Biagi E, Bonini C, Biondi A, Introna M. Cell-based strategies to manage leukemia relapse: efficacy and feasibility of immunotherapy approaches. Leukemia 29(1), 1-10 (2014).

47. Balduzzi A, Di Maio L, Silvestri D et al. Minimal residual disease before and after transplantation for childhood acute lymphoblastic leukaemia: is there any room for intervention? Br. J. Haematol. 164(3), 396-408 (2014).

48. Liu J, Zhang X, Zhong JF, Zhang C. CAR-T cells and allogeneic hematopoietic stem cell transplantation for relapsed/refractory B-cell acute lymphoblastic leukemia. Immunotherapy 9(13), 1115-1125 (2017).

-. Comparatively reviews the role of CAR-T cells and allogeneic hematopoietic stem cell transplantation in relapsed/refractory B-cell acute lymphoblastic leukemia.

49. Nagafuji K, Miyamoto T, Eto T et al. Monitoring of minimal residual disease (MRD) is useful to predict prognosis of adult patients with Ph-negative ALL: results of a prospective study (ALL MRD2002 study). J. Hematol. Oncol. 6, 14 (2013).

50. Chen X, Wen Q, Stucky A et al. Relapse pathway of glioblastoma revealed by single-cell molecular analysis. Carcinogenesis 39(7), 931-936 (2018).

51. Chen Y, Millstein J, Liu Y et al. Single-cell digital lysates generated by phase-switch microfluidic device reveal transcriptome perturbation of cell cycle. ACS Nano 12(5), 4687-4694 (2018).

52. Doubrovina E, Oflaz-Sozmen B, Prockop SE et al. Adoptive immunotherapy with unselected or EBV-specific T cells for biopsy-proven EBV+ lymphomas after allogeneic hematopoietic cell transplantation. Blood 119(11), 2644-2656 (2012).

53. Peggs KS, Verfuerth S, Pizzey A et al. Adoptive cellular therapy for early cytomegalovirus infection after allogeneic stem-cell transplantation with virus-specific T-cell lines. Lancet 362(9393), 1375-1377 (2003).

54. Heslop HE, Slobod KS, Pule MA et al. Long-term outcome of EBV-specific T-cell infusions to prevent or treat EBV-related lymphoproliferative disease in transplant recipients. Blood 15(5), 925-935 (2010).

55. Cruz CR, Micklethwaite KP, Savoldo B et al. Infusion of donor-derived CD19-redirected virus-specific T cells for B-cell malignancies relapsed after allogeneic stem cell transplant: a Phase I study. Blood 122(17), 2965-2973 (2013).

- First to report two patients with CR received preventive infusion of CAR-T cells for refractory/relapsed ALL after allo-HSCT.

56. Kebriaei P, Singh H, Huls MH et al. Phase I trials using Sleeping Beauty to generate CD19-specific CAR T cells. J. Clin. Invest. 126(9), 3363-3376 (2016).

-. The first paper to report larger patients received preventive infusion of CAR-T cells for refractory/relapsed ALL after allo-HSCT. This method is safe and effective. No serious complications are found.

57. Cho JH, Collins JJ, Wong WW. Universal chimeric antigen receptors for multiplexed and logical control of T cell responses. Cell 173(6), 1426-1438 (2018).

-• The first paper to report that CAR-T cells can combat relapse, mitigate overactivation and enhance specificity.

58. Ghassemi S, Nunez-Cruz S, O'Connor RS et al. Reducing ex vivo culture improves the antileukemic activity of chimeric antigen receptor (CAR) T cells. Cancer Immunol. Res. 6(9), 1100-1109 (2018).

-• The first paper to report that producing CAR-T cells with less time can improve effector function. 